\title{
Entre dos tierras y en tierra de nadie: el reflejo del multilingüismo peninsular en la historiografía literaria ibérica ${ }^{1}$
}

\author{
Santiago PÉREZ ISASI \\ Centro de Estudos Comparatistas (Faculdade de Letras) \\ Universidade de Lisboa / Universidad de Deusto \\ santi_perez_isasi@yahoo.es
}

\begin{abstract}
RESUMEN
Este artículo pretende plantear la cuestión de la inadecuación de los esquemas nacionales tradicionalmente aplicados a la configuración de la historia literaria, cuando se proyectan sobre un territorio multilingüe como es la Península Ibérica. Partiremos para ello del estudio de escritores que escapan a las estrictas clasificaciones lingüísticas corrientes, por escribir en diversas lenguas de la Península Ibérica distintas a las consideradas "nacionales", o por escribir en varias lenguas simultáneamente, y analizaremos su tratamiento en las historias de la literatura española y portuguesa durante el siglo XIX.
\end{abstract}

Palabras clave: Historia literaria, literaturas ibéricas, multilingüismo, nacionalismo.

[Recibido, febrero 2012; aprobado, mayo 2012]

In between lands and in no man's land: the reflection of Peninsular multilingual-
ism in Iberian literary history

\begin{abstract}
This article aims at analyzing the question of the inadequacy of the national boundaries traditionally applied in the configuration of literary history, when projected on a multilingual territory like the Iberian Peninsula. The starting point for this reflections will be the study of those Iberian writers who escape the usual linguistic classifications, because they write in languages other than the "national language', or because they write simultaneously in more than one language, and their representation in Spanish and Portuguese literary histories from the 19th century onwards.
\end{abstract}

Keywords: Literary History, Iberian literatures, multilingualism, nationalism.

\footnotetext{
${ }^{1}$ El presente trabajo es resultado del proyecto de investigación posdoctoral "La historiografía romántica de la literatura española en su contexto europeo: traducción, identidad y transnacionalidad", financiado por el Programa de Perfeccionamiento del Personal Investigador del Gobierno Vasco, con adscripción a la Universidad de Deusto (Bilbao) y realizado como investigador del Centro de Estudos Comparatistas de la Universidade de Lisboa, con supervisión de Helena Buescu.
} 
El nacimiento y expansión del nacionalismo entre 1800 y 1918 es uno de los fenómenos más influyentes y definitivos de la historia europea contemporánea: en poco más de 100 años, una ideología inicialmente residual o marginal pasó a convertirse en el principio rector fundamental de las relaciones internacionales, con consecuencias obviamente trascendentales y en ocasiones trágicas. ${ }^{2}$ Como explica paladinamente Joseph Roth en su novela El busto del Emperador: "en el siglo XIX se había descubierto que todo individuo tiene que pertenecer a una nación o a una raza determinadas si realmente pretende ser reconocido como ciudadano burgués" $(2003: 19)^{3}$. Y a esta tarea de descubrir naciones a las que se pudiera pertenecer -a "inventar tradiciones", según la expresión de Hobsbawm y Ranger (2002); a "imaginar comunidades", según la de Anderson (1983)- se dedicó una parte creciente de la intelligentsia europea, en un proyecto sin precedentes, por su extensión y su éxito, de construcción de identidades "desde arriba" a través de instrumentos como los símbolos nacionales (banderas, himnos, escudos), la creación de sistemas nacionales y centralizados de enseñanza o de defensa, o la adecuada reformulación (por no decir abiertamente "manipulación") del pasado y su memoria.

La literatura, y los estudios literarios, también se vieron obviamente afectado por este radical cambio de paradigma en la concepción del mundo, que coincidió además con reformulaciones igualmente radicales en áreas como la Estética, la Poética o la Historia. Ya la primera crítica romántica, encabezada por los hermanos Friedrich y Wilhelm Schlegel, aplicó el concepto herderiano de Volksgeist (el "espíritu del pueblo" que caracteriza la esencia de cada nación) al estudio de la historia literaria, estableciendo firmemente el principio de que cada nación crea una literatura acorde con

\footnotetext{
${ }^{2}$ Estudiosos del nacionalismo como E. Kedourie (1985) o E. Geller (1988) lo consideran como un fenómeno esencialmente negativo en la historia de la humanidad, y como la causa principal de las dos Guerras Mundiales. Es por otra parte común identificar diversas "fases" (temporales y geográficas) en la expansión del nacionalismo por Europa y el mundo. E. H. Carr (1945) distingue tres (o cuatro) periodos solapantes -desde 1800 hasta 1870 ; desde 1870 hasta 1914 y desde 1914 hasta 1939 , con un posible cuarto periodo "post-nacional" tras la Segunda Guerra Mundial-, mientras que Gellner distingue cuatro "zonas de evolución del nacionalismo": Zona 1: los grandes estados dinásticos de España, Portugal, Francia e Inglaterra; Zona 2: Italia y Alemania; Zona 3: los Balcanes, el Cáucaso, el Volga...; Zona 4: la exURSS. (1999: 97-110)

3 J. Leersen explica en términos más académicos el surgimiento del nacionalismo en el tránsito del siglo XVIII al XIX: "nationalism emerges in the nineteenth century from eighteenth-century roots: Herder's belief in the individuality of nations, Rousseau's belief in the sovereignty of the nation, a general discourse of national peculiarities and 'characters'. What changes from the eighteenth century to the nineteenth is this: 1.- an unprecedented imperial campaign mounted by Napoleon and fiercely resented outside France; this turns the eighteenth-century notions of tyranny and liberty from a power imbalance within the state (between rulers and governed) into one of power imbalance between states (between occupier and occupied); 2.- the rise of Romantic idealism which sees national character as a spiritual principle, a 'soul', rather than as a set of peculiarities; 3.- the Romantic belief that a nation's culture, and in particular its language, are the manifestation of its soul and essence; 4.- the historicist belief that all culture must be seen as an organic tradition linking generations across centuries" (2006: 125-6).
} 
su propio carácter, y que por tanto no todas las literaturas pueden ser juzgadas por el mismo patrón crítico, sino estudiadas individual e históricamente. ${ }^{4}$

No es posible, ni tiene sentido a estas alturas, negar el valor que las aportaciones de los hermanos Schlegel y la escuela romántica alemana tuvieron para la renovación de los estudios literarios: entre sus efectos benéficos se cuenta la recuperación crítica de la literatura medieval europea; la revalorización del teatro del Siglo de Oro español, o la reinterpretación, siempre enriquecedora, de autores como Cervantes, Shakespeare o Camões. La propia idea de historia literaria (la narrativización cronológica y, frecuentemente, teleológica, de los hechos literarios de una nación) no habría existido sin esta revisión romántica del concepto de literatura, del concepto de historia o de la relación de ambas entre sí y con la colectividad que las produjo.

Pero tampoco tiene sentido ocultar que la división nacional de las literaturas europeas, así como la identificación entre nación, lengua y literatura, edificó un sistema crítico, historiográfico y epistemológico ${ }^{5}$ que se convirtió en una cama de Procusto a cuya medida fueron recortados o estirados los fenómenos literarios que eran su objeto. No es el menor de estos recortes el olvido -prácticamente definitivo ya- del enorme caudal de literatura europea escrita en latín durante la Edad Media; ni es el menos importante de sus manipulaciones el proyectar hacia pasados remotos divisiones nacionales modernas claramente anacrónicas.

Las literaturas ibéricas no fueron por supuesto una excepción a esta norma. Aunque encuadradas por la crítica romántica en un arco geográfico y cultural común (el Sur latino, católico y romántico de Europa $)^{6}$, sus respectivas literaturas quedaron divididas en capítulos, libros o disciplinas distintas por una frontera infranqueable que separaba rígidamente a los autores de uno y otro país, en virtud de la retroproyección anacrónica de los dos Estados modernos existentes, ya desde las primeras historias de la literatura europea escritas en el siglo XIX. ${ }^{7}$ No solo eso: en el caso de España, además, la identificación progresiva de cada nación con una lengua y una literatura era problemática porque ocultaba, o minusvaloraba como

\footnotetext{
${ }^{4}$ Sobre los inicios de la crítica y la historiografía literaria románticas, y su aplicación en España, véase, entre otros, Behler (1992), Flitter (1993), Romero Tobar (1994) y Saglia (1997).

${ }^{5}$ Sistema que continua fundamentalmente vigente hasta nuestros días, como demuestra la división lingüístico-nacional de los departamentos de Filología o la publicación, todavía en curso, de una nueva Historia de la literatura española (editorial Crítica), bajo dirección de José-Carlos Mainer.

6 "La poesía de los países católicos, la española, la italiana y la portuguesa, forman en ese un conjunto íntimamente unido; por cuya razón las abrazaré con una sola ojeada" (F. Schlegel 1843: II,70). "La poesía de los pueblos meridionales y fieles al catolicismo estaba, en el siglo XVI y aun en el XVII, en una armonía perfecta; por lo menos tenía una marcha absolutamente parecida" (F. Schlegel 1843: II, 131). Vid., a este respecto, Domínguez (2006)

${ }^{7}$ La Geschichte der Poesie und Beredsamkeit seit dem Ende des dreizehnten Jahrhunderts, publicada por Friedrich Bouterwek originalmente entre 1801 y 1819, está unánimemente considerada como la primera historia literaria del siglo XIX; en ella se incluyen libros diferentes dedicados a la literatura española y a la literatura portuguesa, que fueron sin embargo traducidos al inglés conjuntamente.
} 
una anomalía, la existencia de otras lenguas literarias, como el catalán, el gallego, el árabe o el hebreo. ${ }^{8}$

Es evidente, para cualquiera que conozca la historia de las literaturas ibéricas y sus complejas interrelaciones e influencias mutuas, que esta polarización en torno a dos focos independientes y monolingües, si bien es ampliamente productiva en muchos aspectos (de ahí su éxito y su permanencia), es sin embargo incapaz de dar respuesta satisfactoria a casos de autores periféricos o trans-fronterizos, ni de dar cuenta de la compleja red de relaciones de poder, prestigio o fuerza que llevaron a determinados autores a abandonar una lengua y preferir otra para sus creaciones literarias. ${ }^{9}$ En un esquema historiográfico estrictamente nacional (en su sentido más reduccionista), escritores bilingües como Gil Vicente, Juan Boscán o Rosalía de Castro -y muchos otros que ocupan lugares mucho más secundarios en sus respectivos cánones nacionales- están al mismo tiempo entre dos tierras y en tierra de nadie: pertenecen a dos sistemas lingüísticos y por consiguiente a dos historias literarias, pero al mismo tiempo ocupan un lugar incómodo en el gran esquema historiográfico, al poner precisamente en cuestión la idoneidad del propio sistema.

En realidad, el principal problema del modelo historiográfico nacional no es que oculte la existencia de estos fenómenos literarios periféricos o transfronterizos (de hecho, no costará encontrar los nombres de esos autores en las historias de la literatura española o portuguesa), sino el hecho de ignorar que estos fenómenos de bilingüismo o diglosia dentro del sistema literario nacional no son excepcionales ni casuales, sino sistémicos: no responden a mudanzas accidentales de la voluntad individual, sino a modulaciones prolongadas en las relaciones de poder y prestigio entre las lenguas de la Península Ibérica.

Consideremos el primero de los ejemplos en los que me gustaría detenerme, siquiera brevemente: la lírica medieval galaico-portuguesa, y más específicamente las Cantigas, algunas de las cuales son atribuidas al propio rey de Castilla Alfonso

\footnotetext{
${ }^{8}$ En otro lugar (Pérez Isasi, en prensa a) he mostrado cómo esta identificación "una nación, una lengua, una literatura" no fue tan inmediata ni tan indiscutible, sino que fue de hecho acentuándose con el paso del tiempo y de las historias literarias; de hecho, la práctica totalidad de las historias de la literatura española escritas en el siglo XIX incluyen capítulos dedicados a las literaturas catalana o gallega, y existieron intentos (el más notable, el de Amador de los Ríos) de configurar un canon de la literatura española abarcador de las obras producidas en cualquier lengua en territorio español. Sin embargo, la centralidad del castellano es evidente, tanto por extensión como por representatividad en el conjunto de las literaturas "españolas": "Muy difícil es, por no decir imposible, designar con precisión la época en que nació la poesía española, o hablando con más propiedad, castellana" (Ticknor 1851-6: I,15). Incluso Amador de los Ríos, que, como hemos dicho, "Cabe a la España Central, que había adoptado por término de expresión la lengua castellana, la gloria de reflejar más poderosamente y con un fin más general aquella múltiple cultura' (Amador de los Ríos 1861-5:IV, 151)

${ }^{9}$ Hay, por supuesto, una corriente teórica relativamente reciente (y creciente) que aplica un enfoque supranacional como el que proponemos al estudio de las literaturas ibéricas. Señalemos como hitos fundamentales de esta rama la Comparative History of Literatures in the Iberian Peninsula (Cabo Aseguinolaza, Abuin y Domínguez (2010) o, desde una perspectiva teórica y en un contexto distinto (estadounidense en vez de europeo) el alegato en favor de los "Estudios Ibéricos" realizado por J. R: Resina (2009).
} 
$\mathrm{X}$ el Sabio. ${ }^{10} \mathrm{Su}$ mera existencia desafía los límites establecidos por la historia literaria romántica, que proyecta al pasado las fronteras históricamente construidas en los siglos recientes: escritas en el margen del sistema literario ibérico (o en el espacio intermedio entre los dos sistemas literarios ibéricos, si se acepta la división nacional establecida), pero con un gran valor simbólico - por su autor, por su antigüedad, por su propio valor literario-, exigen de los historiadores una ubicación adecuada, una justificación y una explicación satisfactoria.

Porque, de hecho, los historiadores son conscientes del incómodo encaje de las Cantigas en sus esquemas históricos, hasta el punto de que Fitzmaurice-Kelly, ya en la transición al siglo XX, afirma que:

Tales Cantigas, en número de cuatrocientas veinte, poco más o menos, no pertenecen a la literatura castellana, puesto que están escritas en gallego, lengua que han empleado bastantes autores castellanos; pero no podemos dejar enteramente a un lado un monumento de semejante importancia. (FitzmauriceKelly 1901: 40)

Es sin duda significativo que Friedrich Bouterwek, autor de la primera "historia de la literatura española" del siglo XIX, no las mencione cuando repasa la producción del Rey Sabio (de cuya figura, en general, no ofrece una lectura muy favorecedora), mientras que en otros lugares de su Geschichte original sí hace mención a la estrecha relación entre las literaturas española, portuguesa y gallega. ${ }^{11}{ }^{12}$ Por su parte, Simonde de Sismondi, en su Historia de la literatura española (traducción parcial de De la litterature du midi de l'Europe, originalmente publicada en 1813) se limita a afirmar que "se conserva de él en Toledo un manuscrito que contiene unas Cánticas de Nuestra Señora en dialecto gallego" (Sismondi 1841-2: I, 56).

Otros historiadores, como Amador de los Ríos, George Ticknor o James Fitzmaurice-Kelly, que sí mencionan las Cantigas de Alfonso X, se ven en la obligación de señalar su excepcionalidad y de rechazar que su existencia tenga alguna significación más allá de lo anecdótico. Esto sorprende especialmente en el caso de Amador de los Ríos, quien como hemos apuntado ya ofrece una visión lingüística y culturalmente más amplia de la literatura española, y que sin embargo es especial-

\footnotetext{
${ }^{10}$ Para el propósito de este artículo, es indiferente que dichas Cantigas se debieran a la propia mano del rey Alfonso, que fueran compuestas por su círculo poético o que fueran simplemente compuestas a instancia suya.

11 "La antigua poesía castellana estuvo tan unida desde su nacimiento a la portuguesa y la gallega, como ajena se había mantenido a la lemosina" (Bouterwek 1829: 19). Este extracto proviene del prefacio que F. Bouterwek incluyó en su historia (traducida por Carmen Valcárcel Rivera y Santiago Navarro Pastor para la edición de la ed. Verbum), y que fue significativamente excluido por los traductores españoles "por no ser más que una repetición de lo que escribieron Velázquez y Sarmiento" (Bouterwek 1829: 4).

12 "...the Galician and Portuguese languages and poetry were originally, and even after the separation of Portugal from the Castiles, scarcely distinguishable from each other" (Bouterwek 1847: II, 1-2). La parte de la obra de Bouterwek dedicada a la literatura portuguesa nunca fue traducida al español ni al portugués; cito a través de la traducción inglesa, publicada en 1847
} 
mente insistente en separar esta producción gallega del rey Alfonso del núcleo principal y germinal de la poesía castellana, y que defiende con contundencia la primacía temporal de la poesía española en su conjunto:

Y bien será consignar ante todo, que si esta peregrina circunstancia ha podido extraviar la crítica de muy entendidos investigadores, no debe en modo alguno inducirnos al error en que ellos cayeron, apartando lo accidental y transitorio de lo esencial y constante en la poesía y literatura españolas, para deducir y sustentar la verdad histórica. Porque ya fuera el Rey Sabio pasara su infancia y aun su primera juventud en el suelo de Galicia, ya que la misma dulzura del dialecto hablado en aquella comarca le moviese a emplearlo, nunca habrá razón para asentar que fue este el primer instrumento de la poesía española y el único modelo en metros y rimas de la que ha merecido por excelencia el título de castellana. (Amador de los Ríos 1861-5: III, 500)

Más claramente lo expresa aún George Ticknor, quien considera que se trata de una "circunstancia extraordinaria que no es posible explicar satisfactoriamente" (I, 43); y de hecho dedica las siguientes páginas a intentar dar una respuesta, hasta confesar que éstas "son cuestiones que hoy día es imposible dilucidar" (I, 46). Las Cantigas no encajan, como vemos, en un esquema monolingüe y nacional de la literatura española; las estrategias de los historiadores, por lo tanto, pasan por ignorarlas, disminuir su valor relativamente al canon literario nacional, o declararlas un "caso excepcional".

Lo mismo cabría decir de los escritores medievales catalanes o valencianos, ${ }^{13}$ cuya extensa y notable literatura es difícil de ignorar o calificar como "accidente" o reducir a la excepcionalidad; con todo, los escritores en catalán son presentados como una anomalía que requiere ser subsanada y, una vez más, explicada y justificada. Muy interesante es, en este sentido, el caso de Amador de los Ríos, cuya argumentación con respecto a la literatura escrita en catalán consta de dos puntos casi contradictorios: en primer lugar, la afirmación de que la literatura catalana, aunque no esté escrita en castellano, refleja el espíritu español, y por lo tanto es española:

...si hay una época de imitación, en que los poetas catalanes se confunden, aun en el uso de la lengua, con los que nacen y florecen allende los Pirineos, lícito es observar, para gloria del nombre español, que pasado aquel primer momento, imperan en los cantos poéticos de los catalanes la gravedad y la cordura, que formaban la base del carácter nacional, resplandeciendo en ellos al propio tiempo la devoción y el patriotismo. (Amador de los Ríos 1861-5: III, 460)

Y, en segundo lugar, la constatación, casi eufórica, de la tendencia hacia la "castellanización" de la literatura escrita en Cataluña a partir del siglo XV:

\footnotetext{
${ }^{13}$ No tenemos intención de entrar en el debate, más político que lingüístico, sobre la unidad o divergencia entre el catalán y el valenciano; a efectos de la historiografía analizada en este artículo, las producciones de los escritores catalanes, valencianos y mallorquines forman una unidad indistinta, y como tal las trataremos también en este trabajo.
} 
...a pesar de la vitalidad que todavía entrañaba la nacionalidad catalana, y verdes aún los brillantes lauros ganados por sus más celebrados poetas, comienza a recibirse en aquellas regiones, y dentro de su propio parnaso, la influencia de la lengua y del arte, que florecen en la España Central, augurando ya claramente, conforme dejamos advertido, la grande unidad literaria, cuya realización no estaba en verdad muy lejana. (Amador de los Ríos 1861-5: VI, 487)

Esta última afirmación, claramente teleológica (que se podría reformular como la convicción de que era el destino natural de las literaturas hispánicas el centralizarse en torno a Castilla), se manifiesta también, con diversas variantes, en otros historiadores de la literatura española, que ofrecen razones distintas para justificar este abandono literario del catalán en favor del castellano: o bien una supuesta "superioridad lingüística" del segundo, o bien una "superioridad política" del reino de Castilla sobre sus competidores peninsulares:

...la grata lengua de los trovadores [catalán y valenciano] debió de carecer de perfección. De lo contrario, los poetas de Cataluña no se hubieran convertido tan rápidamente en prosélitos de la lengua castellana (Bouterwek 1829:14, nota 3)

...la más poderosa de las monarquías españolas era la de Castilla, que ha recibido en herencia las conquistas, la grandeza y la gloria de los demás estados de la Península, y que por lo mismo merece más atención. (Simonde de Sismondi 1841-2: I, 83)

Es interesante igualmente la postura de George Ticknor, quien en este punto se aparta de sus predecesores, no tanto en la descripción de los hechos, cuanto en su valoración. Así, por un lado afirma que:

La degeneración y envilecimiento de los dos dialectos más cultivados en las provincias del este de España, que comenzó, según hemos visto, en el reinado de los Reyes Católicos, puede decirse que se consumó con el establecimiento de la corte de la monarquía, primeramente en Castilla la Vieja, y después en Castilla la Nueva, pues desde este momento la superior autoridad del castellano quedó finalmente asegurada y reconocida. El cambio no fue por cierto ni injusto ni inoportuno: la lengua del Norte era en aquel tiempo más llena, robusta y rica en idiotismos, y bajo todos los conceptos más apta y adecuada que los dialectos del sur, para ser la lengua nacional. (Ticknor 1851-6: I, 362).

Y sin embargo, a renglón seguido, el hispanista americano no se congratula por este fenómeno, aunque lo considere parte de la evolución natural de la historia lingüística y literaria ibérica, sino que presenta la pérdida de una lengua literaria como un hecho digno de lamentarse: "la decadencia lenta y progresiva, y final pérdida de un idioma, hacen agolpar a la imaginación ideas tristes” (Ticknor 1851-6: I 363). 
En el caso de la lengua y la literatura catalanas, por lo tanto, la estrategia historiográfica es distinta a la aplicada en el caso de las Cantigas: no se trata ya de un ocultar o declarar excepcionales determinados fenómenos literarios, sino que se plantean un conjunto de estrategias textuales de asimilación, en primer lugar declarando que la literatura catalana, pese a su diferencia lingüística, forma parte propiamente de la literatura española por su espíritu esencial (por expresar el Volksgeist hispánico, en otras palabras); y en segundo lugar mediante la celebración más o menos explícita (excepción hecha de Ticknor) de la centralización literaria en torno a Castilla, presentada como un fenómeno no solo natural sino deseable, en virtud de una supuesta superioridad lingüística, política o espiritual. ${ }^{14}$

Antes de acabar, me gustaría apuntar tan solo (tratarlo en profundidad daría material para varias monografías) el caso del tratamiento de los escritores que compusieron una obra bilingüe portugués-español, en algunos casos ocupando lugares importantes en ambos cánones literarios simultáneamente (el caso de Gil Vicente es en este sentido paradigmático $)^{15}$. Es evidente que estos autores también desafían la estricta división nacional de la historia literaria, basada en la separación estricta de las literaturas española y portuguesa. Nuevamente, la existencia de escritores bilingües (españoles que escriben en portugués o, más frecuentemente, portugueses que escriben en castellano) fue tan habitual, que cabe preguntarse si la división de la Península Ibérica en dos historias literarias completamente independientes tiene sentido (¿Por qué dos literaturas exactamente? ¿Por qué no más, o menos?). El propio F. Schlegel afirmaba esta continuidad intrínseca entre ambas:

Solo los Portugueses, que formaban un pueblo y un reino aparte, conservaron en la península su lengua y su poesía particulares; no obstante Portugal continuó teniendo con Castilla un comercio íntimo, cuyo origen subía a una época muy remota: así es que muchos Portugueses escribían en castellano, y una multitud de cosas que se consideran como provenientes de la antigua Castilla derivan sin embargo de aquellos. La poesía de las dos naciones tiene una analogía tan grande, que no es fácil distinguir con respecto a la invención lo que pertenece más a una que a otra. (Schlegel 1843: II, 91)

Es necesario, en este punto, hacer una distinción fundamental, para señalar que la historiografía literaria portuguesa, en su configuración narrativa esencial, se muestra mucho más permeable al reconocimiento de la relación de influencia del vecino, que la española, por lo que los autores que escribieron en español además de en portugués (Luis de Camões o Gil Vicente, por citar solo dos ejemplos suficientemente paradigmáticos) se incardinan en una trama conjunta que los explica, principalmente a través de dos momentos de especial proximidad entre ambos re-

\footnotetext{
${ }^{14}$ Por supuesto, estas ideas pueden considerarse un precedente directo de los planteamientos de Ramón Menéndez Pidal, quien hizo de la centralidad lingüística y literaria de Castilla el eje de su programa filológico.

${ }_{15}$ Precisamente por su carácter paradigmático, la bibliografía sobre Gil Vicente y su bilingüismo literario es ya bastante extensa. Destaquemos, por ejemplo, el volumen coordinado por Fernández García y Pociña López (2004)
} 
inos y sus respectivas literaturas: los tiempos medievales, en que la escisión cultural entre Portugal y España no estaba todavía claramente delimitada, y el periodo de la "Monarquía Dual" (1580-1640), época en que los Reyes Felipe II, Felipe III y Felipe IV gobernaron simultáneamente los territorios de España y Portugal (dominación española que se considera, además, la causa fundamental de la decadencia de las letras portuguesas desde el siglo XVII).

Uma vil adulação havia muitas vezes preferido o idioma estranho ao nosso; o poder monachal e jesuitico censura vergonhosamente todas as obras, chegando a alterar as dos grandes mestres do seculo precedente; e em fim, a inutil crueldade da infame Inquisição, continuando a suffocar os espiritos, extingue até a ultima faísca do fogo patriotico, que animára nossos antigos poetas. (Figueiredo 1844:183)

Esta construcción de una trama que integre a los escritores bilingües o no quiere decir, obviamente, que los escritores bilingües español-portugués, o los escritores portugueses que escribieron única o principalmente en español, encajen sin tropiezos en el esquema historiográfico nacional portugués; no es raro encontrar, efectivamente, ejemplos de historias que deciden excluir a este tipo de autores "no nacionales", como es el caso de la historia de Costa e Silva (1850):

...prescindi n'este Ensaio de mencionar os Poetas, que só escreveram em latin, e em hespanhol, bem que entre estes haja muitos de grande merecimento, e cujas obras possuo, ou tenho visto: mas fallando em rigor, esta falta não deve tornar-se mui sensivel, porque Poetas, que só escreveram em verso Latino, ou Castelhano, posto que nacessem em Portugal, não sam Poetas Portuguezes. (Silva 1850-55: I, 5-6)

En cambio, en la parte española de la frontera no existe una narrativa semejante que vincule a estos escritores (portugueses, pero que escribieron en español) ni entre sí, ni con el conjunto de causas o circunstancias que motivaron su condición de escritores transfronterizos o transculturales. La aparición frecuente, en las historias de la literatura portuguesa, de nombres como Sá de Miranda, Gil Vicente, Francisco Manuel de Melo o Camões (normalmente con la grafía extendida "Camoens") no se integra en una trama ni constituye un sentido: son apariciones esporádicas, individuales y no se relacionan con su contexto. Así, por ejemplo, Simonde de Sismondi se limita a señalar que "Saa de Miranda... pertenece más bien a la literatura portuguesa que a la española" (Sismondi 1841-2: I, 218), y Gil de Zárate dice de Francisco Manuel de Melo, que es "el más célebre de todos los portugueses que escribieron en español" (Gil de Zárate 1844: I, 217). La ausencia de un marco conceptual o narrativo en el que integrar estas apariciones subrepticias de escritores portugueses en la historia literaria española solo puede causar desconcierto y desasosiego en los historiadores que deben lidiar con ellas. Es el caso, muy evidente, de George Ticknor, quien no acierta a explicarse por qué Gil Vicente pudo decidir escribir una parte de sus obras en español y no en portugués: 
Lo primero que llama atención en las composiciones de Gil Vicente, es su forma enteramente española, y el estar la mayor parte escritas en idioma castellano. [...] Por qué razón Gil Vicente adoptó este método, no es fácil atinar; las dos lenguas tienen sin duda alguna mucha afinidad, y los escritores de una y de otra nación, particularmente los portugueses, se han distinguido muy a menudo en el uso de ambas; si bien estos no han querido nunca conceder que la suya fuese ni menos rica, ni menos apta para todo género de composición que la de sus vecinos y rivales. Quizá se deba en este caso a la circunstancia de que las cortes de Castilla y Portugal estaban a la sazón estrechamente unidas por dobles casamientos; a que el rey D. Manuel llevaba continuamente consigo truhanes y juglares castellanos que le divertían; a que la Reina, esposa de este, era española; o finalmente, a que Gil Vicente creyó deber imitar en esto, como en otras muchas cosas, a su maestro Juan del Encina. (Ticknor 1851-6: 297-8)

Una adecuada comprensión del significado histórico de la existencia de estos escritores bilingües (tanto en el lado español como en el portugués) exigiría integrarlos en un discurso único, complejo y necesariamente multicultural, que sobrepasase las fronteras nacionales establecidas y que contemplase el papel que un mismo escritor bilingüe puede desempeñar, simultáneamente, en diversos subsistemas de la literatura ibérica. En su formulación romántica y post-romántica más habitual, la historia literaria (sobre todo en el lado español de la frontera) muestra a estos escritores o bien escindidos de su otra mitad, o bien como fenómenos casi inexplicables, estrellas fugaces multilingües en un cosmos esencialmente monolingüe y estático.

En definitiva, la historia literaria romántica, apoyada en los nacientes sistemas educativos y universitarios nacionales, y sus permutaciones hasta prácticamente nuestros días, perpetuaron una visión excesivamente compartimentada y estática del sistema literario europeo: ocultaron completamente la existencia de determinados fenómenos literarios, y fallaron en su intento de explicar y relacionar otros. En el caso que nos ocupa, no son meros accidentes o anécdotas hechos como que un rey castellano escriba poesías en gallego; que los poetas valencianos abandonen el catalán en sus producciones; o que en el Madrid o la Lisboa de la Monarquía Dual se representasen obras de dramaturgos portugueses escritas en español. En todos los casos se trata de manifestaciones de complejas relaciones de prestigio y poder (simbólico en unos casos, efectivo en otros), que una historia literaria centrada en una única lengua difícilmente explicará. Solo una perspectiva comparatista y, concretamente, ibérica, puede construir una historia diferente, que dé cuenta de las fluctuaciones, continuidades y rupturas de esas relaciones de poder, y sus consecuencias para la literatura. Y esa es una historia que aún está por escribir. 


\section{Referencias bibliográficas}

AMADOR DE LOS RÍOS, José (1861-5): Historia Crítica de la literatura española. Madrid: Imprenta de José Rodríguez.

ANDERSON, Benedict (1983): Imagined Communities. Londres: Verso.

BEHLER, Ernst (1993): German Romantic Literary Theory. Cambridge: Cambridge University Press.

FIGUEIREDO, Antonio Cardoso Borges de (1844): Bosquejo histórico da Litteratura Classica, Grega, Latina e Portugueza, para uso das Escholas. Coimbra: Imprensa da Universidade.

BOUTERWEK, Friedrich (1829 [2002]): Historia de la literatura española. Madrid: Verbum.

- (1847) History of Spanish literature, Londres: David Vogue.

CABO ASEGUINOLAZA, Fernando (2001): "National Canon Formation as Interliterary Process. The Spanish Case". Literary Research/Recherche littéraire vol. 18, 35 (primavera-otoño): 51-62.

CABO ASEGUINOLAZA, Fernando; ABUIN, Anxo y DOMÍNGUEZ, César (eds.) (2010): A Comparative History of Literatures in the Iberian Peninsula. Amsterdam/Philadelphia: John Benjamins, vol I.

SILVA, José María da Costa e (1850-55): Ensaio biographico-critico sobre os melhores poetas portuguezes. Lisboa: Imprensa Silviana, 10 tomos, 5 vols.

DOMÍNGUEZ, César (2006): "The South European Orient: A Comparative Reflection on Space in Literary History", Modern Language Quarterly 67 (4): 419-449.

FERNÁNDEZ GARCÍA, María Jesús y POCIÑA LÓPEZ, Andrés José (2004): Gil Vicente, clásico luso-español. Mérida: Junta de Extremadura.

FITZMAURICE-KELLY, James (1901): Historia de la literatura española. Madrid: Librería general de Victoriano Suárez.

FLITTER, Derek (1992): Spanish Romantic Literary Theory and Criticism. Cambridge: Cambridge University Press.

GELLNER, Ernst (1988): Naciones y nacionalismo. Madrid: Alianza. - (1999): Nacionalismo. Barcelona: Destino.

GIL DE ZÁRATE, Antonio (1844): Resumen histórico de la literatura española. Madrid: Boix, 3 vols.

HOBSBAWM, Eric y RANGER, Terence (2002): La invención de la tradición. Barcelona: Crítica.

KEDOURIE, Elie (1985): Nacionalismo. Madrid: Centro de Estudios Constitucionales.

PÉREZ ISASI, (2011): "La historia literaria como herramienta de nacionalización en España”, Oihenart. Cuadernos de Lengua y Literatura 25: 267-179.

- (en prensa a): "The limits of Spanishness in Spanish 19th-century literary history", Bulletin of Hispanic Studies, University of Liverpool.

- (en prensa b): "Presencia y ausencia de lo vasco en las historias de la literatura española (1800-1939)", Oihenart, Cuadernos de Lengua y Literatura. 
RESINA, Joan Ramon (2009): Del hispanismo a los estudios ibéricos. Una propuesta federativa para el ámbito cultural. Madrid: Biblioteca Nueva.

ROMERO TOBAR, Leonardo (1994): Panorama crítico del romanticismo español. Madrid: Castalia.

ROTH, Joseph (2003): El busto del Emperador. Barcelona: Acantilado.

SAGLIA, Diego (1997): “The True Essence of Romanticism: Romantic Theories of Spain and the Question of Spanish Romanticism". Journal of Iberian and Latin American Studies, vol. 3, 2: 127-145.

SCHLEGEL, Friedrich (1843): Historia de la literatura antigua y moderna. Barcelona: Libr. de J. Oliveres y Gavarró.

SIMONDE DE SISMONDI, Jean-Charles-Leonard (1841-2): Historia de la literatura española. Sevilla: Imprenta de Álvarez y Compañía.

TICKNOR, George (1851-56): Historia de la literatura española. Madrid: Imprenta de la Publicidad. 